\title{
Compact ultra-fast vertical nanopositioner for improving scanning probe microscope scan speed
}

\author{
Brian J. Kenton, ${ }^{1}$ Andrew J. Fleming, ${ }^{2, a)}$ and Kam K. Leang ${ }^{1, b)}$ \\ ${ }^{1}$ Department of Mechanical Engineering, University of Nevada Reno, Reno, Nevada 89557-0312, USA \\ ${ }^{2}$ School of Electrical Engineering and Computer Science, University of Newcastle, NSW 2308, Australia
}

(Received 5 April 2011; accepted 31 October 2011; published online 13 December 2011)

\begin{abstract}
The mechanical design of a high-bandwidth, short-range vertical positioning stage is described for integration with a commercial scanning probe microscope (SPM) for dual-stage actuation to significantly improve scanning performance. The vertical motion of the sample platform is driven by a stiff and compact piezo-stack actuator and guided by a novel circular flexure to minimize undesirable mechanical resonances that can limit the performance of the vertical feedback control loop. Finite element analysis is performed to study the key issues that affect performance. To relax the need for properly securing the stage to a working surface, such as a laboratory workbench, an inertial cancellation scheme is utilized. The measured dominant unloaded mechanical resonance of a prototype stage is above $150 \mathrm{kHz}$ and the travel range is approximately $1.56 \mu \mathrm{m}$. The high-bandwidth stage is experimentally evaluated with a basic commercial SPM, and results show over 25-times improvement in the scanning performance. () 2011 American Institute of Physics. [doi:10.1063/1.3664613]
\end{abstract}

\section{INTRODUCTION}

Scanning probe microscopes (SPMs), such as atomic force microscopes (AFMs), enable precise control, manipulation, and interrogation of matter at the nanoscale. ${ }^{1}$ Recent advances in mechanical design, ${ }^{2,3}$ drive electronics, ${ }^{4}$ data acquisition hardware and software, ${ }^{5}$ and control algorithms ${ }^{6}$ have lead to the development of high-speed nanopositioning systems for applications that include video-rate SPMs. ${ }^{3,7,8}$ Despite significant improvement in the lateral scanning bandwidth, one of the major limitations is the relatively slow response of the vertical positioning stage due to tradeoffs between travel range and response time. Recent attention has focused on improving the vertical $(z)$ positioning bandwidth for better control of the tip-to-sample interaction. . $^{9} 11,12$

In the majority of commercial SPMs, the tip-to-sample interaction along the $z$-direction is controlled by a piezoelectric actuator, such as a tube-shaped actuator. ${ }^{13}$ Tube-shaped piezoactuators are commonly used because they are compact and offer relatively large travel range that can exceed $10 \mu \mathrm{m}$; however, the low mechanical resonance frequencies and significant cross-coupling behavior limit performance. ${ }^{14}$

A novel high-speed vertical positioning stage is presented which can be retrofitted to most top-down, scan-by-probe style commercial SPMs to provide at least an order of magnitude improvement in the control of the tip-to-sample interaction. The design employs a compact and stiff piezo-stack actuator coupled with a circular flexure to minimize off-axis resonance frequencies that can jeopardize the performance of the vertical feedback loop. A detailed finite element analysis of the dynamic performance of the vertical positioner is presented, where the key challenges that affect performance are described. To relax the need for properly securing the stage,

a)Electronic mail: Andrew.Fleming@ @ewcastle.edu.au.

b) Author to whom correspondence should be addressed. Electronic mail: kam@unr.edu. for example, to a laboratory workbench, an inertial cancellation scheme is utilized and experimentally evaluated. The vertical positioner is integrated with a basic commercial AFM to demonstrate over 25-times improvement in scanning performance. It is noted that similar stages have recently been proposed and applied in SPMs, ${ }^{9,10}$ but this work focuses on the details of the mechanical design from a practical point of view, as well as the implementation of the stage with a commercial SPM.

\section{MECHANICAL DESIGN}

\section{A. Dual-stage actuation for SPMs}

Dual-stage actuation, which combines long-range, lowspeed positioning and short-range, fast actuation, has been proposed to overcome the tradeoff between travel range and positioning bandwidth. ${ }^{9,11,12,15,16}$ Figure 1 shows the block diagram of a dual-stage SPM system. The SPM's existing vertical positioning stage (denoted by LSA for low-speed actuator), which has slow-to-moderate response time, is used for positioning over large range, while a separate short-range actuator (denoted by HSA for high-speed actuator) with the ability for fast movement makes up for the slow response of the long-range actuator. The overall control objective is to regulate the cantilever deflection, that is, to maintain a constant reference deflection $\delta_{\text {ref }}$ or tapping-mode amplitude set by the user. As illustrated in Fig. 1, the deflection error signal (e) is applied to a controller, $C(s)$, and the output of the controller splits into complimentary filters: a low-pass filter $L(s)$ for the low-speed actuator and a high-pass filter $H(s)$ for the high-speed actuator. The dynamics of the piezo-amplifiers are represented by $A_{L}(s)$ and $A_{H}(s)$, which are assumed to be sufficiently high so that they can be approximated by a static gain. Images can be obtained by plotting the control input $u$ as a function of the lateral scan position, for example. 


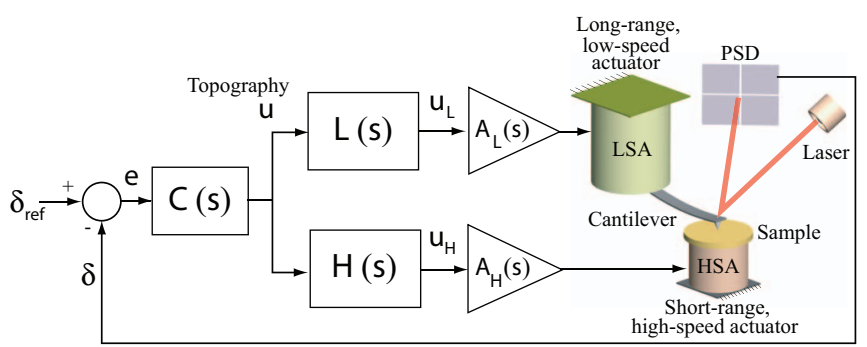

FIG. 1. (Color online) Block diagram of dual-stage SPM system.

\section{B. Optimizing the mechanical response}

The desired dominant resonance frequency of the highspeed stage when loaded with a sample mass (approximately $2 \mathrm{~g}$ ) should be at least $100 \mathrm{kHz}$. This value is chosen because it will enable between ten- to one-hundred-times increase in the bandwidth of a standard SPM. Additionally, this choice allows for the high-speed stage to be applied to video-rate SPM where fast vertical positioning is crucial. ${ }^{9}$ The required travel range is 1 to $2 \mu \mathrm{m}$, and thus, compact plate- or ringstacks are the preferred choice because of their relatively high mechanical resonance frequency, low capacitance, and sufficient travel range. For example, a commercially available 2-mm-thick plate-stack actuator with a cross section of $5 \mathrm{~mm}$ $\times 5 \mathrm{~mm}$ (e.g., Noliac CAMP07) has a nominal capacitance of $100 \mathrm{nF}$, a free stroke of $3.1 \mu \mathrm{m}$, and an unloaded resonance frequency greater than $500 \mathrm{kHz}$. The same plate-stack actuator is available (e.g., Noliac SCMAP07) with ceramic end caps for electrical isolation and offers $2.9 \mu \mathrm{m}$ of travel with an unloaded resonance frequency greater than $250 \mathrm{kHz}$ (total actuator length of $4 \mathrm{~mm}$ ). In practice, the effects of boundary conditions, sample mass, and end-caps reduce both the travel range and mechanical resonance frequency.

Figure 2 offers several possible end constraints for using plate- or ring-stack piezoactuators for vertical positioning in SPM. The appropriate choice, however, depends on the size of the sample mass and the geometry of the actuator. The simplest and most common arrangement is the fixed-free situation illustrated in Fig. 2(a), where $m$ represents the sample mass. Unfortunately, this configuration may not be ideal for fast vertical positioning for several reasons. First, if the total

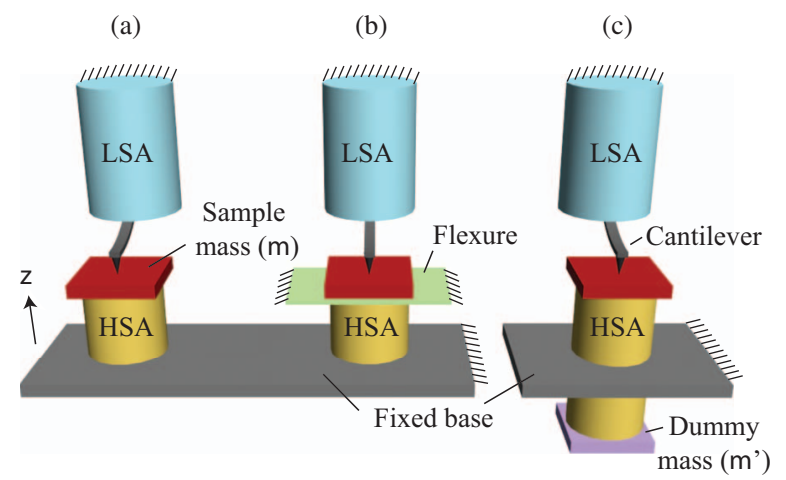

FIG. 2. (Color online) Mounting configurations for plate-stack piezoactuator with sample mass $m$ for dual-stage vertical positioning: (a) fixed-free, (b) fixed and free end flexure guided, and (c) inertial cancellation with dummy mass $m^{\prime}$. The actuation direction is denoted by $z$. length of the actuator including the sample mass is relatively long compared to its transverse dimensions, the first mechanical resonance is likely a bending mode, causing the sample to displace side-to-side as well as up and down, when excited. The bending mode can cause significant distortion in AFM imaging and results in a resonance pole in the transfer function between the piezo input voltage and the AFM cantilever deflection. If the pole contributes a significant phase delay, it can limit the bandwidth of the vertical feedback controller.

As an approximation, let $h$ denote the overall length of the actuator (which includes the end caps for electrical isolation) and $t$ be the sample thickness. Then, the first bending resonance frequency for the piezoactuator and sample mass with fixed-free end constraints is given by ${ }^{17}$

$$
f_{x}=\frac{1}{2 \pi}\left(\frac{1.875}{h+t}\right)^{2} \sqrt{\frac{E_{e} I}{\rho_{e} A}} \mathrm{~Hz},
$$

where $E_{e}$ and $\rho_{e}$ are the effective Young's modulus ${ }^{18}$ and apparent density, respectively, of the composite piezo-stack actuator, end caps, and sample mass; $I$ is the moment of inertia; and $A$ is the cross-sectional area. For the same boundary conditions, the first longitudinal resonance frequency of the actuator is approximated by

$$
f_{z}=\frac{1}{4(h+t)} \sqrt{\frac{E_{e}}{\rho_{e}}} \mathrm{~Hz} .
$$

For a 4-mm-long plate-stack piezoactuator with cross section $5 \mathrm{~mm} \times 5 \mathrm{~mm}$, using $E_{e}=33 \mathrm{GPa}, \rho_{e}=7800 \mathrm{~kg} / \mathrm{m}^{3}$, and a sample mass of thickness $t=1 \mathrm{~mm}$, the first bending and longitudinal resonance frequencies are estimated as $66 \mathrm{kHz}$ and $103 \mathrm{kHz}$, respectively. The finite element analysis (FEA) method is used to determine the modal results to compare to the values estimated by Eqs. (1) and (2), and the results are presented in Fig. 3, first row (a). The first bending and piston (longitudinal) resonance frequencies using FEA are $43 \mathrm{kHz}$ and $103 \mathrm{kHz}$, respectively. The large discrepancy between the bending resonance frequencies is due to Eq. (1) being derived for a long slender beam, neglecting the effects of shear. However, the trend in Eqs. (1) and (2) is consistent with the FEA results in that the bending mode appears before, and at a much lower frequency than, the longitudinal (actuation) mode. To increase the mechanical resonance frequency, two options are considered: (i) reducing the overall length of the actuator and (ii) using a compliant flexure to increase the stiffness of the actuator in the transverse directions ( $x$ and $y$ ). These two options are explored in more detail in the following.

Making the actuator more compact will increase the mechanical resonance, but the tradeoff is reduced travel range. The 4-mm-long actuator considered above consists of a 2-mm-thick plate-stack actuator sandwiched between two 1-mm-thick ceramic end caps. As recommended by the manufacturer, the end caps provide electrical isolation when the actuator is attached to a conducting substrate. The actuator length is reduced by replacing the $1-\mathrm{mm}$ ceramic end caps with custom end caps fabricated from 0.6-mm-thick alumina plate $(E=300 \mathrm{GPa})$. In this case, the new actuator length is reduced by $20 \%$ to $3.2 \mathrm{~mm}$, and the modulus of the end caps is increased by nearly 10 times. The predicted bending 


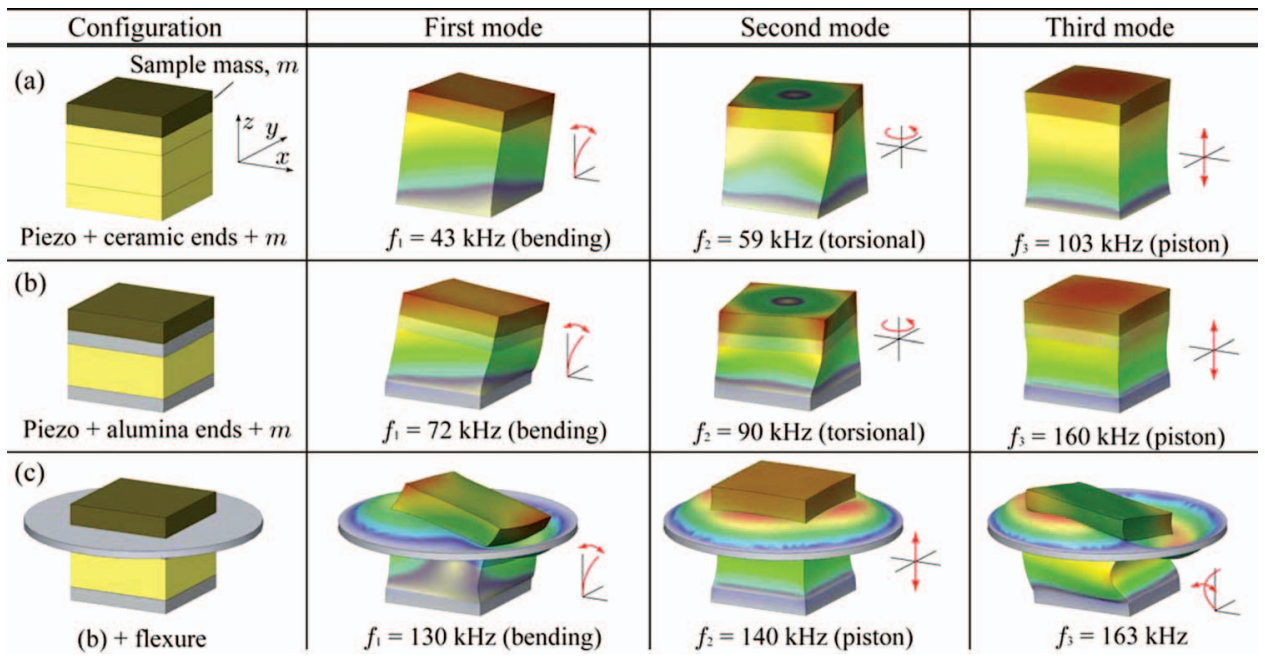

FIG. 3. (Color online) Finite element analysis results for different stage designs involving a piezo-stack actuator: (a) stock 4-mm-tall actuator with sample mass; (b) stock 2-mm-thick actuator with alumina end caps and sample mass; (c) configuration (b) with circular flexure.

and longitudinal resonance frequencies using Eqs. (1) and (2), with $E_{e}=49 \mathrm{GPa}$, are $115 \mathrm{kHz}$ and $149 \mathrm{kHz}$, respectively. The FEA results for this case are shown in Fig. 3, second row (b), where the bending and piston modes show up at $72 \mathrm{kHz}$ and $160 \mathrm{kHz}$, respectively. As predicted, by reducing the overall actuator length and increasing the effective stiffness, the resonance frequencies are increased significantly, with similar trends as the previous case involving the ordering of the bending and longitudinal resonance frequencies.

\section{Circular flexure design}

To minimize the effects of the bending mode, a circular plate flexure is proposed to (i) increase the stiffness of the actuator in the traverse directions ( $x$ and $y$ ) and (ii) guide the movement of the sample mass along the $z$ direction [see Fig. 3, row (c)].

The flexure stiffness $\left(k_{f}\right)$ along the actuation direction is chosen to be less than $10 \%$ of the actuator stiffness to minimize the effect on the stroke of the piezoactuator. ${ }^{19}$ The candidate ring- or plate-stack piezoactuators have stiffness of approximately $385 \mathrm{~N} / \mu \mathrm{m}$, so a stiffness value of less than $38.5 \mathrm{~N} / \mu \mathrm{m}$ is desirable. The flexure material chosen is stainless steel $(E=200 \mathrm{GPa})$, with thickness $t$ of $0.254 \mathrm{~mm}$ (0.010 in.). The required outside radius $r_{a}$ of the flexure is determined as follows.

Consider the circular plate flexure shown in Fig. 4(a) with its outside edge clamped. For convenience, a ring-shaped piezoactuator is attached to the center of the flexure, where the actuator exerts a pressure $P$ on the flexure. The total applied force is $F=P A$, where $A$ is the contact area between the piezoactuator and flexure. The vertical deformation of a circular flexure at the outer edge of the piezoactuator $\left(r_{b}\right)$ due to the applied load $F$ with an ideal guided constraint at $r_{b}$ is ${ }^{20}$

$$
\delta_{z}=\frac{F r_{a}^{3}}{2 \pi r_{b} C_{1}}\left(\frac{C_{2} C_{3}}{C_{4}}-C_{5}\right),
$$

where the constants $C_{j}$, for $j=1,2, \ldots, 5$, are

$$
\begin{aligned}
C_{1} & =\frac{E t^{3}}{12\left(1-v^{2}\right)}, \\
C_{2} & =\frac{1}{4}\left[1-\left(\frac{r_{b}}{r_{a}}\right)^{2}\left(1+2 \ln \left(\frac{r_{a}}{r_{b}}\right)\right)\right], \\
C_{3} & =\frac{r_{b}}{4 r_{a}}\left[\left(\frac{r_{b}}{r_{a}}\right)^{2}-1+2 \ln \left(\frac{r_{a}}{r_{b}}\right)\right], \\
C_{4} & =\frac{1}{2}\left[1-\left(\frac{r_{b}}{r_{a}}\right)^{2}\right], \\
C_{5} & =\frac{r_{b}}{4 r_{a}}\left\{\left[\left(\frac{r_{b}}{r_{a}}\right)^{2}+1\right] \ln \left(\frac{r_{a}}{r_{b}}\right)+\left(\frac{r_{b}}{r_{a}}\right)^{2}-1\right\} .
\end{aligned}
$$

$v$ is Poisson's ratio, $r_{a}$ is the radius of the outside constraint, and $r_{b}$ is the radius of the center constraint. The stiffness of
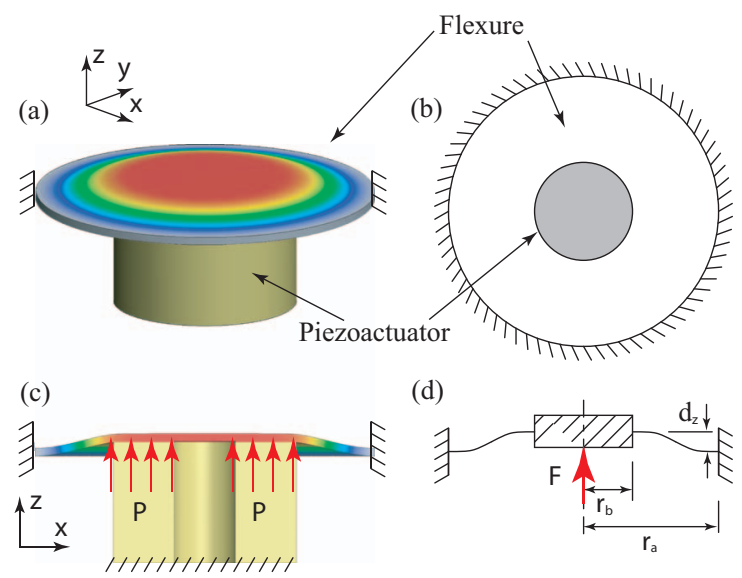

(d)

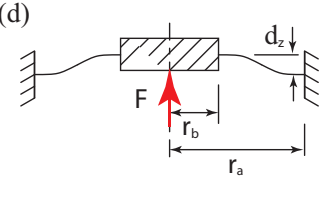

FIG. 4. (Color online) Circular plate flexure for $z$-axis stage. (a) Trimetric, (b) top, and (c) side views, where $P$ denotes the applied pressure; (d) schematic of deformed flexure and loading conditions, where $w$ is the radially distributed load at $r_{o}$. 
the flexure along the actuation direction $z$ is

$$
k_{f}=\frac{F}{\delta_{z}}=\frac{2 \pi r_{b} C_{1}}{r_{a}^{3}}\left(\frac{C_{2} C_{3}}{C_{4}}-C_{5}\right)^{-1} .
$$

The above stiffness equation is then used to determine the outside radius of the flexure for a piezoactuator with radius $r_{b}=3 \mathrm{~mm}$ and flexure of thickness $t=0.254 \mathrm{~mm}$. The solid line in Fig. 5 represents the analytical stiffness $k_{f}$ as a function of flexure radius $r_{a}$. It is shown that the flexure stiffness decreases drastically as the flexure radius increases. FEA results for an ideal guided center constraint are compared to the analytical results and denoted in Fig. 5 by "*." The FEA results follow the same trend as the analytical results and are in good agreement. Specifically, the error is $3.68 \%$ for radius $r_{a}=10 \mathrm{~mm}$ and increases to $16.72 \%$ for $r_{a}=5 \mathrm{~mm}$. FEA is then used to determine the resultant flexure stiffness when the ideal guided boundary condition is replaced with a piezoactuator pushing on the surface of a plate as shown in Fig. 4. The results (denoted by "o" in Fig. 5) show the same stiffness trend while the expected decrease is due to the compliance and slip of the piezoactuator acting on the plate flexure.

To decrease the cost of the piezoactuator, a square plate stack is preferred over a ring-stack. Finite element analysis is used to determine the flexure stiffness when using a $5 \mathrm{~mm}$ $\times 5 \mathrm{~mm}$ square plate-stack with an alumina end plate. The resultant stiffness, depicted by " $X$ " in Fig. 5, shows increased stiffness compared to the ring-stack constraint. This is likely due to the higher modulus alumina end cap and the protruding corners of the square constraint. An outside radius of $5.5 \mathrm{~mm}$ is used to produce a flexure with stiffness of approximately $5 \mathrm{~N} / \mu \mathrm{m}$ for the experiments.

\section{Inertial cancellation}

When a relatively large sample mass is attached to the stage, inertial forces can induce undesirable effects, especially at high speed. ${ }^{7}$ If the stage body is not properly secured to the

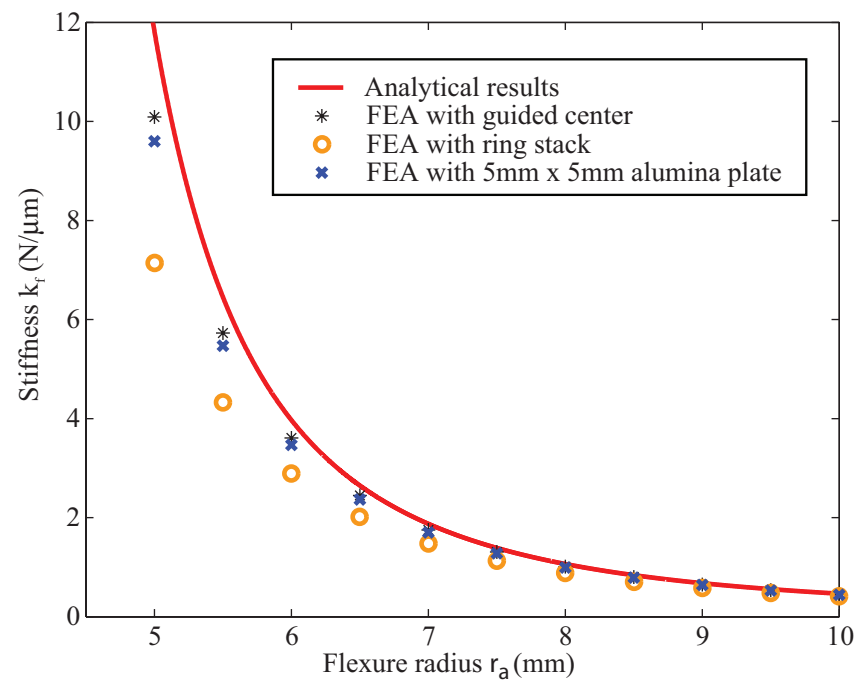

FIG. 5. (Color online) Comparison of analytical and FEA results for stiffness values of circular plate flexure with different radii. working surface, such as a laboratory workbench, the effects can be worse. Additionally, the movement of the piezoactuator can generate acoustic waves which reflect off adjacent supports and fixtures causing additional unwanted vibrations. ${ }^{15}$ Nearby bodies can be made from the same material as the actuator to maximize energy absorption, thus minimizing acoustic wave effects. But for better flexibility and portability, an inertial cancellation scheme in the form of two opposing actuators should be considered to compensate for inertial effects or acoustic disturbances. ${ }^{7}$ The inertial cancellation design shown Fig. 2(c) employs a mirror image of the single piezoactuator and sample mass. For optimum performance, the dummy mass is chosen equal to the mass of the sample. The two piezoactuators are wired in parallel, thus both actuators expand and contract simultaneously; however, the masses displace in opposite directions. For improved performance, circular flexures are added to the ends of the piezoactuators to guide the motion along the actuation direction.

\section{EXPERIMENTAL RESULTS AND DISCUSSION}

\section{A. Manufacturing and assembly}

The mechanical assembly of the vertical stage with inertia cancellation is shown in Fig. 6. The stage body is machined from stainless steel. Two 2 -mm-thick piezo-stack actuators with alumina end caps (each 0.6-mm thick) for electrical isolation are used. The alumina end caps are first epoxied to the piezo-stack actuator and then the whole unit and circular plate flexure are finally glued under pressure in the machined pockets of the stage body.

Because the stage is capable of operating at high-speed, it is critical to create the piezoamplifier with sufficient bandwidth and output power to drive the capacitive piezoelectric load. The nominal capacitance of one single piezo-stack actuator is $100 \mathrm{nF}$. The piezo-amplifiers are built around the Power Amp Design PAD129 power op-amp, with a gain bandwidth product of $1 \mathrm{MHz}$ and typical slew rate of $37 \mathrm{~V} / \mu \mathrm{s}$. The dc power supply for the power op-amp is constructed from two linear regulated 100 V, 3 A supplies (Acopian A100HT300) wired in series to create a 0 to $200 \mathrm{~V}$ supply for the power

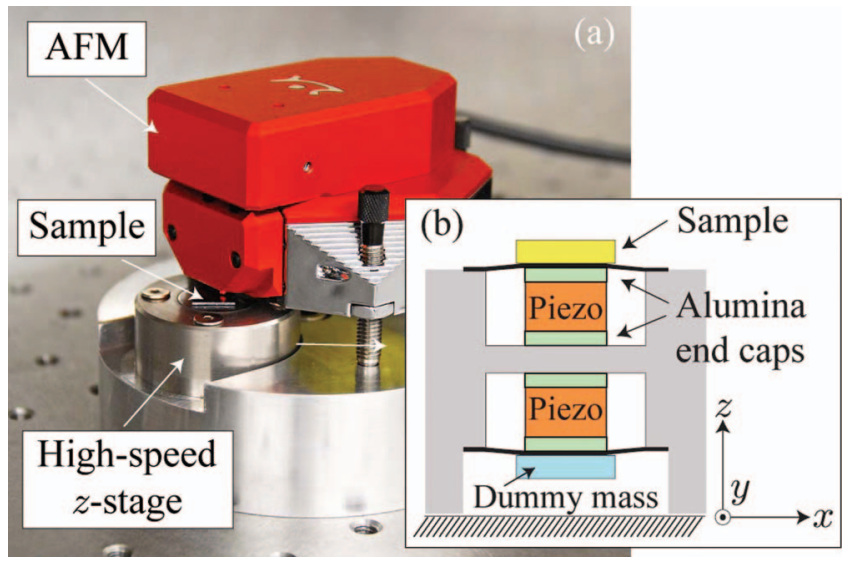

FIG. 6. (Color online) (a) The prototype high-speed $z$-stage integrated with a commercial scan-by-probe AFM. (b) Assembly drawing of the inertial cancellation design. 
(a)

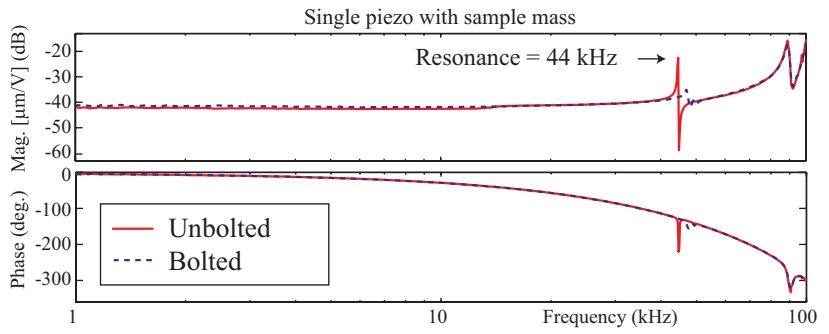

(b)

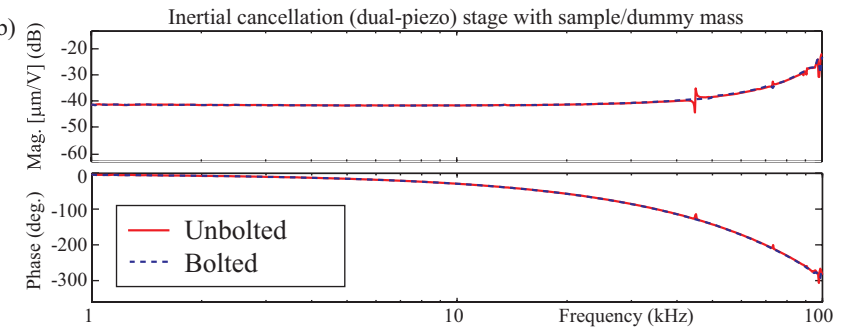

FIG. 7. (Color online) Frequency response functions comparing (a) single piezo design to (b) inertial cancellation (dual piezo) design.

op-amp. Alternatively, PiezoDrive (www.piezodrive.com) low-noise, high-speed piezoactuator amplifiers can be used.

\section{B. Dynamic response and range}

The frequency response functions (FRFs) of the vertical stage are measured using a dynamic signal analyzer (Stanford Research Systems model SRT785) by the swept-sine method by applying a chirp signal created with MATLAB software. The latter approach is used to obtain the response above $102 \mathrm{kHz}$ due to the limitations of the digital signal analyzer (frequency range from dc to $102 \mathrm{kHz}$ ). A single-point laser vibrometer (Polytec CLV-1000 with CLV-800 laser and CLV700 sensor head) is used to measure the velocity of the stage in the vertical direction. The vibrometer has a bandwidth of $250 \mathrm{kHz}$ and is positioned directly above the stage using a tripod. A high-speed data acquisition card (National Instruments PCI-6115, maximum input-output bandwidth of $2.6 \mathrm{MS} / \mathrm{s}$ ) and custom LabView program are used to apply the chirp signal while simultaneously recording the output of the laser vibrometer.

The inertial cancellation design and the effects of securing the stage to the working surface, such as an optical table, are first investigated. For the single-piezo design, two pole-zero pairs (resonance and antiresonance) appear in the frequency response at approximately $44 \mathrm{kHz}$ and $90 \mathrm{kHz}$, as shown in Fig. 7(a). In particular, the magnitude of the resonance frequency near $44 \mathrm{kHz}$ is more pronounced when the stage is not bolted (unsecured) to the optical table. Interestingly, these measured resonance frequencies did not appear in the FEA simulations due to the fact that the base of the stage is ideally constrained. By fastening the stage to the working surface using stainless steel machine screws, the magnitude of the low-frequency resonance is reduced, but shifted to approximately $47 \mathrm{kHz}$ (Fig. 7(a), dashed line). The measured FRFs for the single-piezo design suggest that by not securing the stage to the working surface causes undesirable resonance frequencies. In practice, the $44 \mathrm{kHz}$ resonance frequency can cause significant phase drop which can ultimately limit the vertical control bandwidth.

The FRFs for the inertial cancellation design [Fig. 6] are shown in Fig. 7(b). It is apparent that the inertial cancellation design suppresses the undesirable resonances. When the inertial cancellation stage is secured to the working surface (Fig. 7(b), dashed line), both resonance and anti-resonance frequency pairs which appeared in the FRF of the single-piezo design (Fig. 7(b) dashed line) are not present. The detrimental pole-zero pair is believed to occur due to inertial loads causing the stage body to rattle and rock when the body is not secured to the optical table. By securing the stage body and using inertial cancellation, this behavior is minimized. In fact, the FRF shows evidence of a first dominant mode above $100 \mathrm{kHz}$ as predicted by FEA. It is pointed out that even with the stage body not secured to the working surface, the FRFs shows minimal effect of the detrimental pole-zero pair.

Figure 8 shows the measured FRFs for the unloaded (without sample mass, dashed line) and loaded (with sample mass, solid line) inertial cancellation stage over an extended frequency range ( 5 to $400 \mathrm{kHz}$ ). The unloaded stage shows the first dominant resonance frequency at approximately $156 \mathrm{kHz}$. When loaded with a sample mass, the resonance frequency reduces to approximately $120 \mathrm{kHz}$, which is in good agreement with the FEA results shown in Fig. 3, row (c).

The travel range of the positioner is measured with an ADE capacitive sensor (ADE 2805 sensor with ADE $4810 \mathrm{~S}$ module). With an input of $190 \mathrm{~V}$, the maximum travel range is $1.56 \mu \mathrm{m}$, resulting in a measured sensitivity of $8.22 \mathrm{~nm} / \mathrm{V}$.

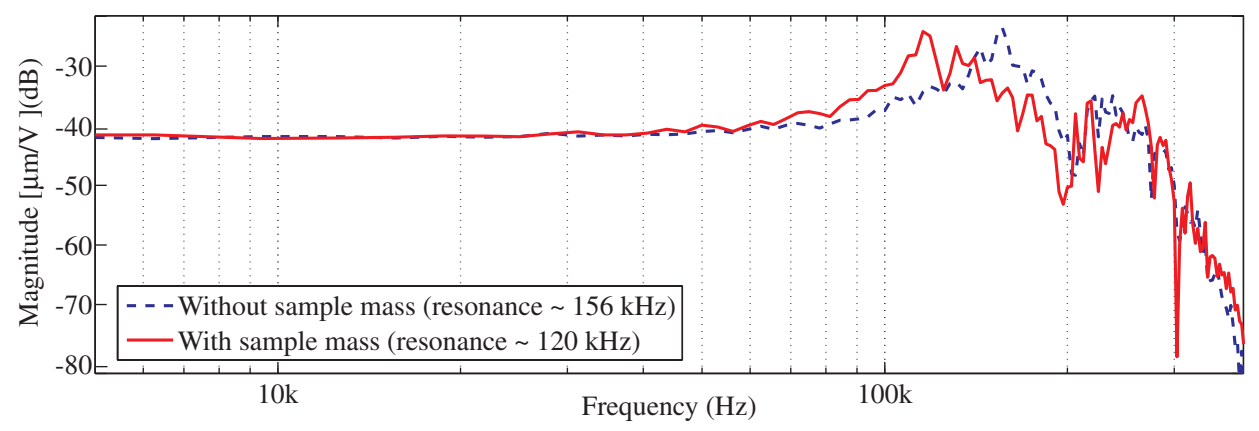

FIG. 8. (Color online) Frequency response of inertial cancellation $z$-stage with 1-mm-thick sample and dummy mass. 
Deflection

(a1)
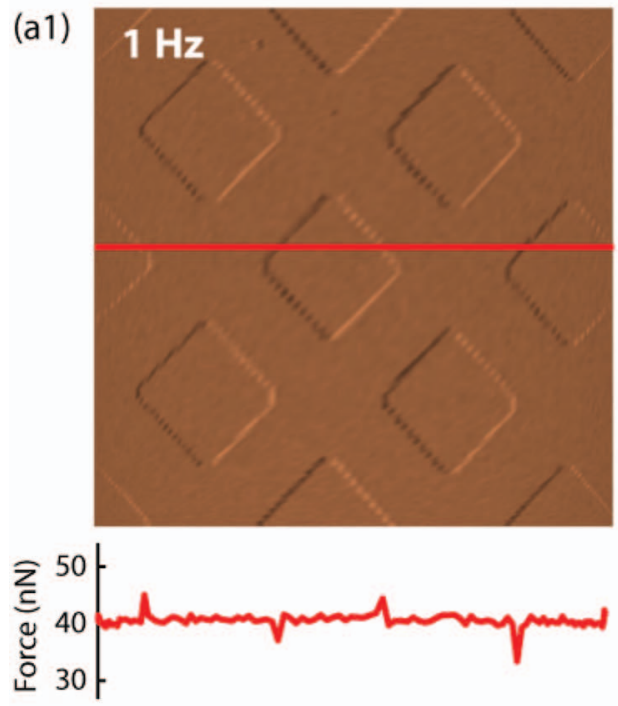

(b1)
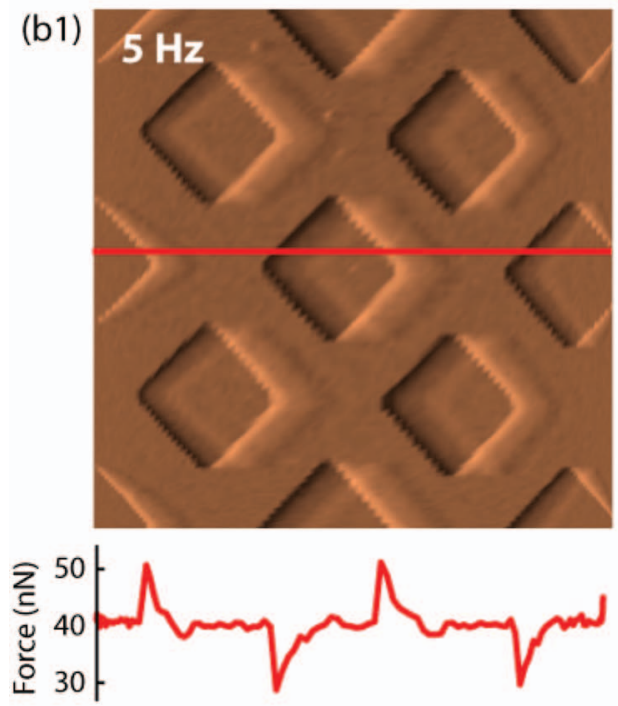

(c1)
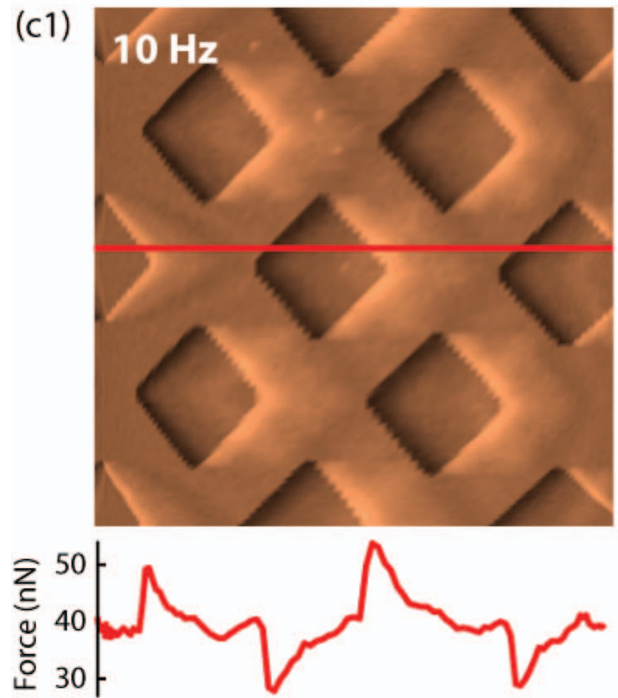

Topography

(a2)
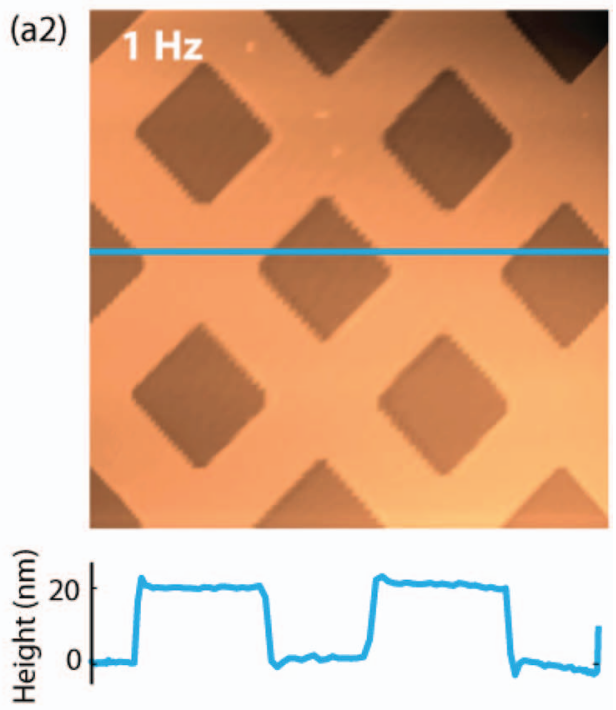

(b2)
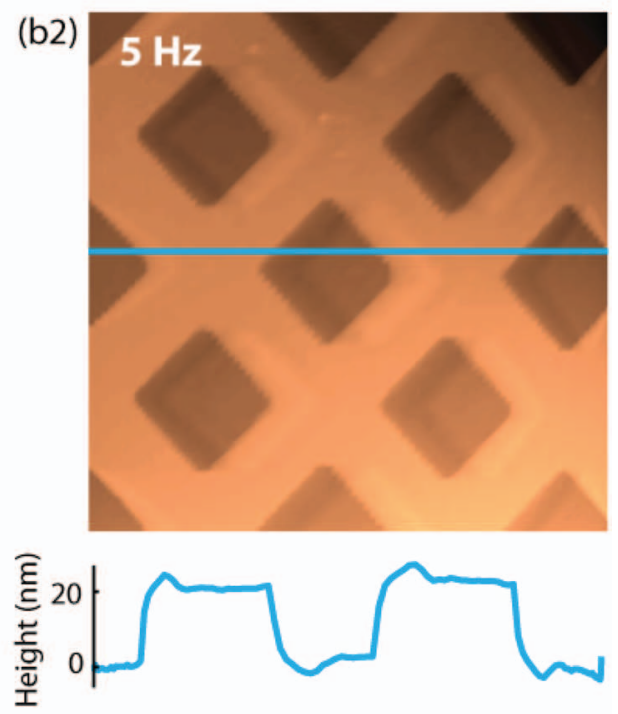

(c2)
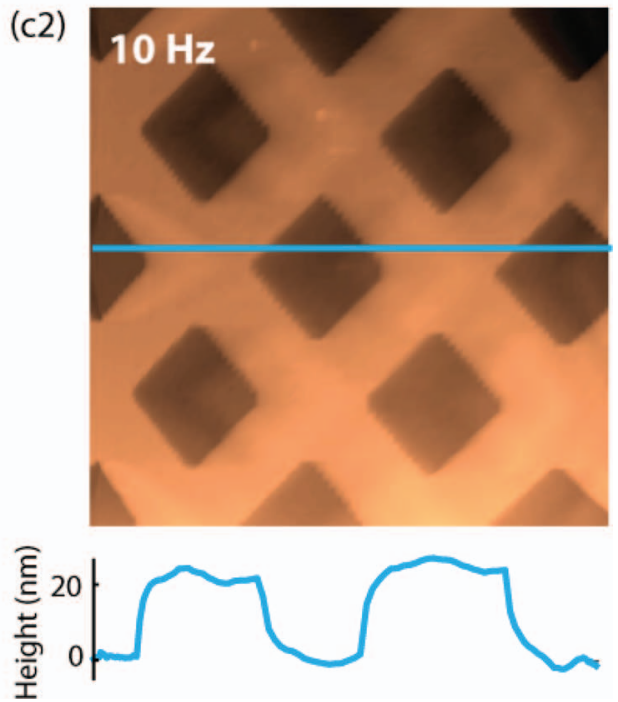

FIG. 9. (Color online) (a1)-(c2) Imaging results for commercial system only. 
Deflection
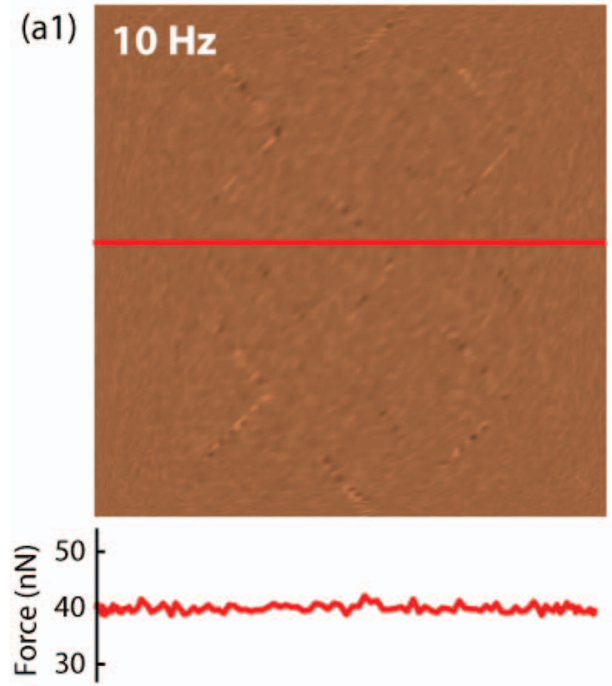

(b1)
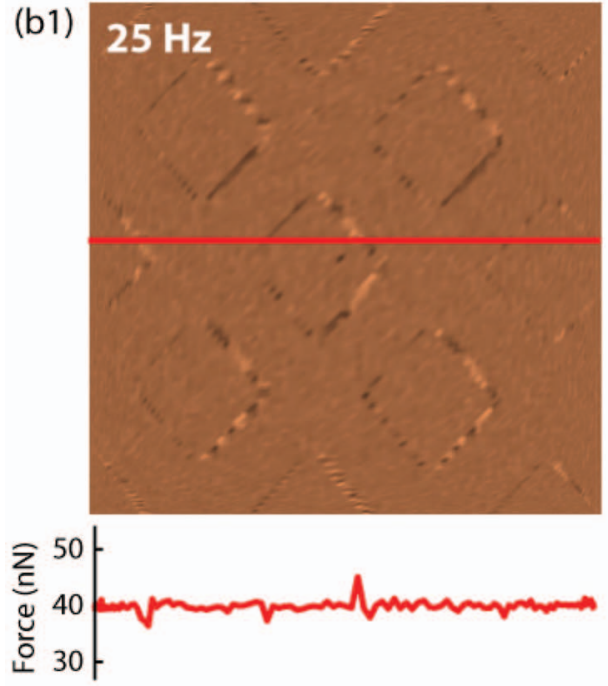

(c1)
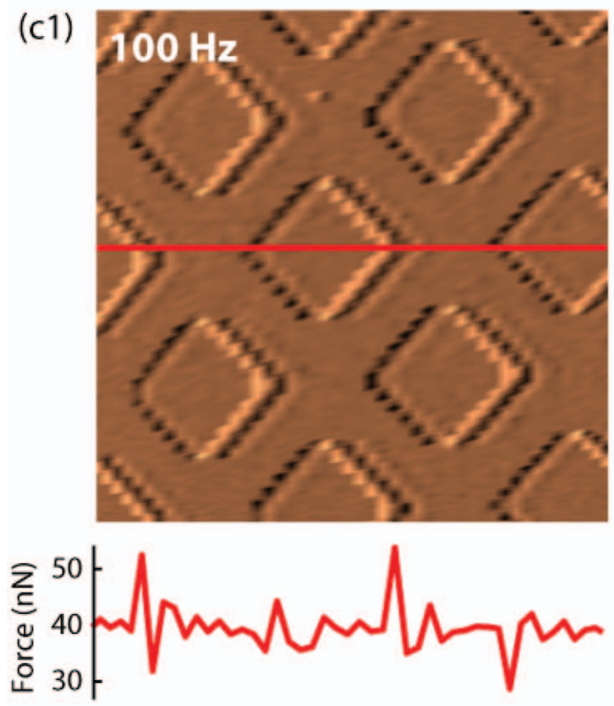

\section{Topography}

(a2)
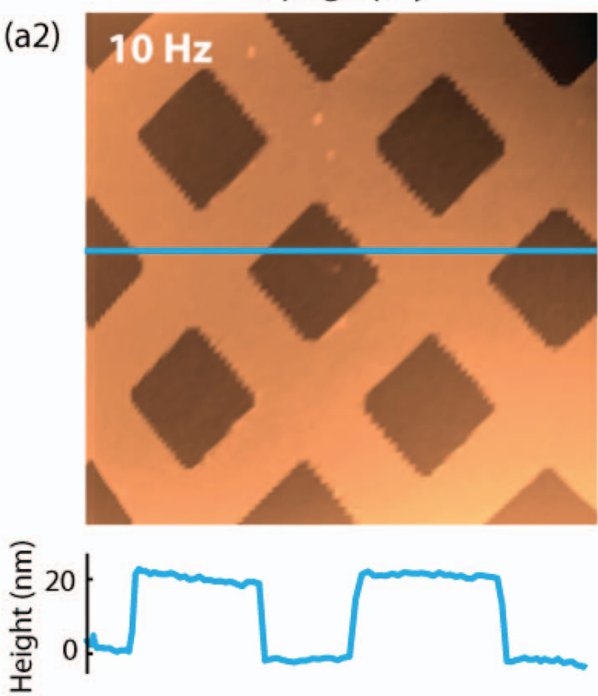

(b2)
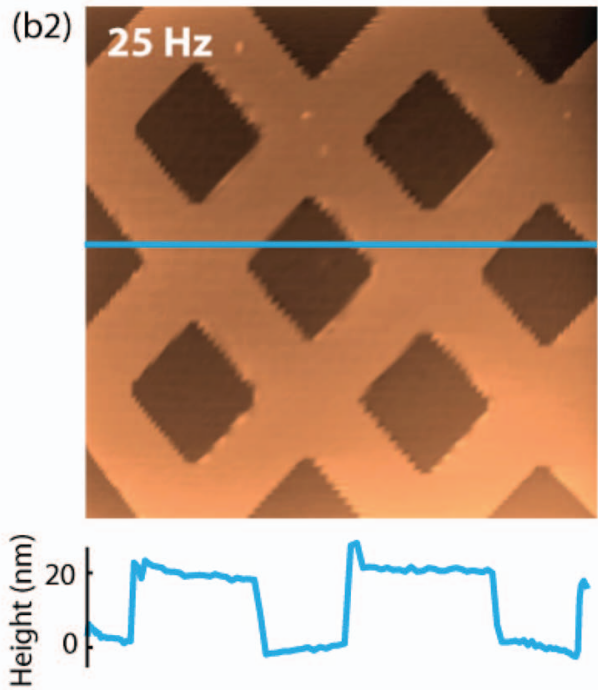

(c2)
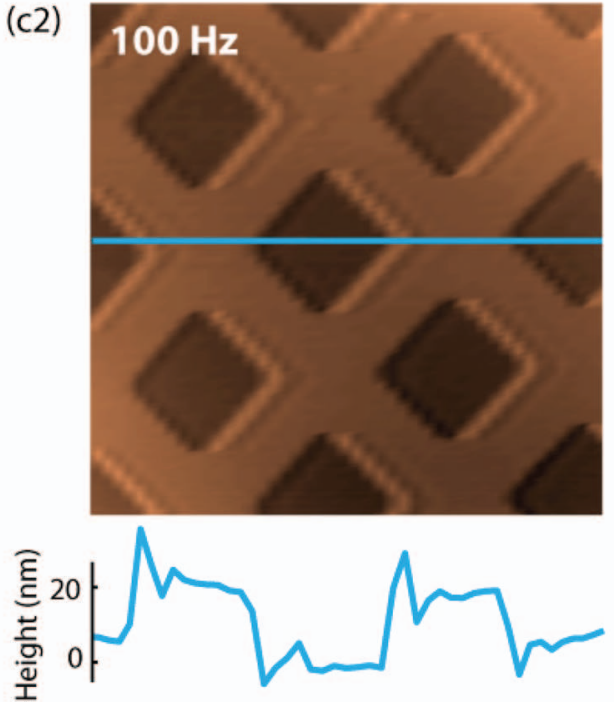

FIG. 10. (Color online) (a1)-(c2) Imaging results for commercial system combined with high-speed vertical stage. 
TABLE I. Cantilever force regulation versus scan frequency.

\begin{tabular}{lccccc}
\hline \hline & \multicolumn{2}{c}{ Low-speed actuator } & & \multicolumn{2}{c}{ High-speed actuator } \\
\cline { 2 - 3 } \cline { 5 - 6 } $\begin{array}{l}\text { Frequency } \\
(\mathrm{Hz})\end{array}$ & $\begin{array}{l}e_{\max } \\
(\mathrm{nN})\end{array}$ & $\begin{array}{l}e_{\mathrm{rms}} \\
(\mathrm{nN})\end{array}$ & & $\begin{array}{l}e_{\max } \\
(\mathrm{nN})\end{array}$ & $\begin{array}{l}e_{\mathrm{rms}} \\
(\mathrm{nN})\end{array}$ \\
\hline 1 & 7.15 & 1.05 & 1.94 & 0.60 \\
5 & 11.64 & 2.87 & 1.30 & 0.50 \\
10 & 14.55 & 4.27 & 2.24 & 0.63 \\
25 & 21.32 & 8.85 & 5.16 & 0.86 \\
50 & $\ldots$ & $\ldots$ & 7.87 & 1.39 \\
75 & $\ldots$ & $\ldots$ & 9.38 & 2.26 \\
100 & $\ldots$ & $\ldots$ & 13.67 & 5.05 \\
\hline \hline
\end{tabular}

\section{AFM imaging results and discussion}

The fabricated high-speed vertical stage is integrated with a commercial scan-by-probe AFM system (NanoSurf easyScan 2) to demonstrate improvement in constant-force, contact-mode imaging of a calibration sample. Figure 6(a) shows a photograph of the AFM and high-speed $z$-stage. A calibration sample with 20 -nm-tall features with pitch of $10 \mu \mathrm{m}$ is attached to the top of the $z$-stage using a thin layer of cyanoacrylate-based fast-acting adhesive. The cantilever is a Vista Probes T190 with a nominal spring constant of $48 \mathrm{~N} / \mathrm{m}$ and measured first resonance frequency at $173 \mathrm{kHz}$.

The first experiment evaluates the performance of the commercial AFM system itself and the imaging results are shown in Fig. 9. For constant force imaging, the reference force is set to $40 \mathrm{nN}$, while the proportional and integral gains are set to 1000 and 10000 using the $z$-controller settings in the AFM software. For a scan rate of $1 \mathrm{~Hz}$, the cantilever deflection is minimized yielding maximum and root-mean-squared error values of $7.15 \mathrm{nN}$ and $1.05 \mathrm{nN}$, respectively (Table I). Variations in cantilever force are depicted in Fig. 9(a1) as dark and light lines along the edges of the surface features. The image features that are lighter in color are areas of increased force [as shown in the trace plot below (a1)] caused by the sample features. The dark features are areas of low force. As the scanning speed increases, the bandwidth of the commercial vertical stage cannot maintain a constant force, causing significant error and image distortion. The upper limit of the scanning frequency for the commercial system is approximately $1 \mathrm{~Hz}$.

In the second experiment, the high-speed vertical positioner is used in conjunction with the AFM's vertical positioning stage. The AFM's existing vertical stage because of its low bandwidth (resonance frequency of $170 \mathrm{~Hz}$ ) is unable to track abrupt sample features at high probe-tip speed as shown above. Additionally, it is noted that at high line rates, the dynamic lateral-to-vertical cross-coupling in the AFM induces vertical displacements greater than the range of the high-speed stage. For this reason, a feedforward input is applied to compensate for the vertical cross-coupling of the AFM, enabling the high-speed stage to track the abrupt surface features. The feedforward input is determined by measuring the lateral-to-vertical cross-coupling (vertical displacement) without the high-speed stage active for a sinusoidal lat- eral scan. From the measured data, an additional control input was generated for the low-speed stage to compensate for the cross-coupling. The improvement in force regulation is readily apparent when the high-speed actuator is added to the system. A dramatic increase in topographical image fidelity is observed while using the short-range, high-speed vertical positioner (see images in Fig. 10). Table I summarizes the maximum and root-mean-squared variation of the force response. The results for the force variation for the AFM combined with the high-speed vertical positioner compared to the stand-alone AFM show increased line rates of over 25 times, underscoring the benefits of the dual-stage system.

\section{CONCLUSIONS}

A high-bandwidth, short-range vertical positioning stage was described that can be integrated with a commercial SPM for dual-stage actuation to significantly improve performance. A compact piezo-stack actuator was used to displace the sample mass, and a novel circular flexure guided the motion of the sample platform to minimize undesirable resonance frequencies that can limit the performance of the vertical feedback control loop. The measured dominant unloaded mechanical resonance frequency of the vertical positioner was above $150 \mathrm{kHz}$ and the travel range was approximately $1.56 \mu \mathrm{m}$. The high-bandwidth stage was experimentally evaluated with a commercial atomic force microscope (AFM), and results showed over 25-times improvement in the scanning performance compared to the commercial vertical stage.

${ }^{1}$ S. M. Salapaka and M. V. Salapaka, IEEE Control Syst. Mag. 28, 65 (2008). ${ }^{2}$ G. Schitter, K. J. Astrom, B. E. DeMartini, P. J. Thurner, K. L. Turner, and P. K. Hansma, IEEE Trans. Control Syst. Technol. 15, 906 (2007).

${ }^{3}$ B. J. Kenton and K. K. Leang, "Design and control of a three-axis serialkinematic high-bandwidth nanopositioner" IEEE/ASME Trans. Mechatron. (in press).

${ }^{4}$ A. J. Fleming, Rev. Sci. Instrum. 80, 104701 (2009).

${ }^{5}$ G. E. Fantner, P. Hegarty, J. H. Kindt, G. Schitter, G. A. G. Cidade, and P. K. Hansma, Rev. Sci. Instrum. 76, 026118 (2005).

${ }^{6}$ S. Devasia, E. Eleftheriou, and S. O. R. Moheimani, IEEE Trans. Control Syst. Technol. 15, 802 (2007).

${ }^{7}$ T. Ando, T. Uchihashi, and T. Fukuma, Prog. Surf. Sci. 83, 337 (2008).

${ }^{8}$ G. Schitter and M. J. Rost, Mater. Today 11, 40 (2008).

${ }^{9} \mathrm{G}$. Schitter, W. F. Rijkee, and N. Phan, in Proceedings of the IEEE Conference on Decision and Control, Cancun, Mexico, 2008 (IEEE, Boston, MA, 2008), p. 5176.

${ }^{10}$ A. J. Fleming, B. J. Kenton, and K. K. Leang, Ultramicroscopy 110, 1205 (2010).

${ }^{11}$ G. Schitter, Tech. Mess. 76, 266 (2009).

${ }^{12}$ A. J. Fleming, IEEE Trans. Control Syst. Technol. 19, 156 (2011).

${ }^{13}$ G. Binnig and D. P. E. Smith, Rev. Sci. Instrum. 57, 1688 (1986).

${ }^{14}$ A. J. Fleming and A. G. Wills, IEEE Trans. Control Syst. Technol. 13, 552 (2009).

${ }^{15}$ D. Knebel, M. Amrein, K. Voigt, and R. Reichelt, Scanning 19, 264 (1997).

${ }^{16}$ K. E. Rifai, O. M. E. Rifai, and K. Youcef-Toumi, in Proceedings of the American Control Conference, Boston, MA, 2004 (IEEE, Boston, MA, 2004), p. 3128.

${ }^{17}$ D. Inman, Engineering Vibration, 2nd ed., (Prentice Hall, Upper Saddle River, NJ, 2001).

${ }^{18}$ E. H. F. Date, J. Phys. D: Appl. Phys. 3, 778 (1970).

${ }^{19}$ Piezo Nano Positionoing: Inspirations, Physik Instruments, Auburn, MA, 2009.

${ }^{20}$ W. C. Young and R. G. Budynas, Roark's Formula for Stress and Strain, 7th ed. (McGraw-Hill, New York, 2002). 